\title{
ANÁLISE DE CARCINOMAS MAMÁRIOS BILATERAIS: UM PERFIL DAS PACIENTES DE UM SERVIÇO DE REFERÊNCIA
}

Camila Vitola Pasetto1, Lucas Roskamp Budel1, Bruno Ribeiro Batista', Mariana de Nadai Andreoli1, Vinicius Milani Budel ${ }^{1}$

${ }^{1}$ Universidade Federal do Paraná - Curitiba (PR), Brazil.

Introduction: Bilateral breast cancer (BBC) is a rare clinical entity. This pattern of neoplasia can be considered synchronous (simultaneous) or metachronous (1 month to 1 year later). Objective: To select cases of BBC patients seen at HC-UFPR and to recognize in these patients clinical and family characteristics, histological and immunohistochemical patterns, and incidences of synchronous/metachronous occurrences. Method: An observational and analytical study of BBC cases of patients treated at HC-UFPR, from January 2003 to October 2019, based on the analysis of medical records, was developed. Result: 42 patients with $\mathrm{BBC}$ were selected and 4 patients were excluded from the study due to incomplete information in the medical record. The incidence of BBC in the surveyed period was 3.64\%. All patients are women with a mean age of 51.82 years. White ethnicity is the most prevalent one ( $82 \%)$. With regard to menopausal status, $42 \%$ of the subjects are pre-menopausal and $58 \%$, post-menopausal. Regarding parity, only $16 \%$ were nulliparous. Half of the patients have a positive family history for neoplasms, with breast cancer present in $46 \%$, ovarian cancer in $16 \%$, and other topography in 68\%. In this sample, the synchronous tumor was present in 55\% of the patients and the metachronous tumor in $45 \%$. Regarding the patients' initial clinical staging, $61 \%$ presented with locally advanced tumor at the first consultation. In the group of synchronous tumors, the ductal type was the most frequent one (93\%), followed by the lobular type (7\%). Regarding immunohistochemical subtypes, patients had Luminal B tumors (43\%), followed by HER (29\%), Triple negative (24\%), and Luminal A (5\%). Comparing the immunohistochemical profile in both tumors, $62 \%$ were in agreement and 48\%, in disagreement. In the group of metachronous tumors, the mean time between the diagnosis of the first tumor and that of the second tumor was 5.68 years. The most common histological type was ductal carcinoma (73\%), followed by lobular carcinoma (11\%), medullary carcinoma (9\%), and metaplastics (7\%). Regarding the immunohistochemical profile, the most present was Luminal B in 32\%, Luminal A in 29\%, Triple negative in 24\%, and HER 2 in 15\%. The immunohistochemical profile was consistent in only $29 \%$ of the patients. Conclusion: In this sample, BBC is associated with relevant family history, with a pattern of presentation, synchronous; frequently, ductal is histological and Luminal B is immunohistochemistry. 